\title{
EL COLOR EN LA MEMORIA: TINTES VEGETALES USADOS EN LA TRADICIÓN DE LAS COMUNIDADES ANDINAS Y AMAZÓNICAS PERUANAS
}

\section{COLOR IN THE MEMORY: PLANT DYES USED IN THE TRADITIONS OF ANDEAN AND AMAZONIAN PERUVIAN COMMUNITIES}

\author{
Joaquina Albán-Castillo ${ }^{1}$, Geraldine Espinoza ${ }^{2}$, Rosario Rojas ${ }^{3}$ y Camilo Díaz Santibáñez ${ }^{2}$
}

\begin{abstract}
Resumen
En el Perú, son escasas las comunidades que aún conservan en su memoria la sabiduría ancestral sobre las plantas y técnicas tintóreas. Esta erosión cultural se debe, sobre todo, a la globalización y la no valorización de los conocimientos tradicionales, los cuales, si no se registran adecuadamente, corren el riesgo de perderse y extinguirse de la identidad étnica. El estudio reconstruye las técnicas tintóreas tradicionales usadas en cuatro comunidades andinas y tres amazónicas, para su revalorización, preservación y uso en la artesanía e industria textil contemporánea. La metodología propuesta incluye la evaluación etnobotánica de plantas con potencial tintóreo y la replicación in situ de los colores obtenidos. El proceso de teñido consta de cuatro fases, ejecutadas de manera consecutiva. Como paso previo a la obtención del tinte, la preparación de la fibra es fundamental en el acabado del producto. Se experimentó con 51 especies vegetales, utilizadas por los pobladores de estas comunidades, incluidas en 30 familias botánicas y 45 géneros. La familia Asteraceae registra el mayor número de incidencias de uso como tintóreo, seguida por la familia Fabaceae. Se observaron diferencias en el tratamiento de las fibras empleadas, dependiendo de su origen animal o vegetal. Se concluye que el uso de plantas tintóreas en comunidades andinas y amazónicas peruanas, aún se conserva en el contexto de su cultura tradicional.

Palabras clave: Andes, Amazonía, etnobotánica, plantas tintóreas, Perú.
\end{abstract}

\begin{abstract}
In Peru, there are few communities that still preserve in their memory the ancestral wisdom on plants and dyeing techniques. This cultural erosion is mainly due to globalization, and the nonvalorization of traditional knowledge, which is not recorded properly, running the risk of losing and extinguishing ethnic identity. This study reconstructs the traditional plant dyeing techniques, used in four Andean and three Amazonian communities, enabling us to reassess, preserve and use them in crafts and contemporary textile industry. The proposed methodology includes the ethnobotanical evaluation of plants with dyeing potential, and the in situ replication of the obtained colors. The dyeing process consists of four steps, which are delivered consecutively. As a step prior to obtaining the dye, the preparation of the fibers is fundamental in the finishing of the product. We tested 51 plant species used by the inhabitants of these communities, including 30 botanical families and 45 genera. The Asteraceae family registers the highest incidence of use as dyes, followed by the Fabaceae family. Differences were observed in the treatment of the fibers used, depending on their animal or plant origin. We concluded that the use of dyeing plants in Peruvian Andean and Amazonian communities is still being preserved, in the context of its traditional culture.
\end{abstract}

Key words: Andes, Amazon, dye plants, ethnobotany, Peru.

\section{Introducción.}

El conocimiento sobre el uso de plantas tintóreas en el Perú es de larga trayectoria histórica, en especial en los aspectos vinculados a su uso en el teñido de textiles (Antúnez de Mayolo, 1989; Brack, 1999; Contreras, 2009; Hurtado \& Rojas, 2005; Rojas R. et al., 2016). Las culturas pre-incas son reconocidas por sus textiles y técnicas usadas para el teñido de fibras a partir del uso de recursos naturales. Estos conocimientos transgeneracionales fueron transmitidos horizontalmente (Zumbuhl, 1979), y debido a que fueron materiales perecibles, resulta difícil encontrar evidencias arqueológicas que permitan identificar las especies empleadas (Ivic \& Berger 2008). En la actualidad, a pesar del descubrimiento de los colorantes sintéticos, como la mauveina (Sing, 1997), aún se conservan las técnicas empleadas desde la antigüedad asociadas al uso de plantas tintóreas.

En América latina, el Caribe y Asia, el uso de recursos forestales no maderables ha sido registrado pertinentemente, evidenciándose que los saberes locales respecto a la obtención y uso de tintes naturales 
aún se conservan (Jansen \& Cardon, 2005; Junsongduang et al., 2017; Paván et al., 2017; Poma, 2016; Trilla et al., 2007).

En el Perú existen algunas iniciativas que intentan recuperar y salvaguardar el patrimonio cultural textil del Perú; como, por ejemplo, el Programa de Reconstrucción de Tecnología Andina (PRETECA) promovido por la ONG Círculo Amigos de la Cultura en asociación con el Instituto Yachay Wasi; así como la iniciativa del Centro de Textiles Tradicionales en el Cusco, las cuales buscan preservar las técnicas textiles que aún se practican en ciertas comunidades andinas. Estos programas requieren del respaldo científico que valide las especies botánicas utilizadas y las técnicas de tinción, para su efectiva puesta en valor.

El estudio tuvo como objetivos recuperar los conocimientos y técnicas tintóreas en base a plantas, a través de su registro y sistematización, como parte del proyecto "Reconstrucción de las técnicas tintóreas precolombinas, en base a productos naturales, para su preservación, revalorización y uso en la artesanía e industria textil contemporánea" (Convenio 130FINCyT-IA-2013). Este se llevó a cabo de acuerdo con los protocolos establecidos en la investigación etnobotánica y teniendo en cuenta el respeto a la propiedad intelectual de los participantes y protección de las especies. Los resultados obtenidos nos han permitido identificar las especies vegetales y técnicas tintóreas que aún persisten en la memoria de los habitantes de las comunidades andinas y amazónicas peruanas.

\section{Materiales y métodos.}

Área de estudio.

El área geográfica en la que se realizó el trabajo de recolección de información y recolección botánica comprende siete localidades ubicadas en las regiones de Loreto (comunidades amazónicas), Ancash y Cusco (comunidades andinas) (Tabla 1, Figura 1). El estudio se ejecutó entre los años 2014-2016.

Recolección Botánica.

En el proceso de la colecta de los ejemplares botánicos, se aplicó el método convencional de recolección, prensado y secado para la posterior determinación taxonómica (Cerrate, 1969; Lima dos Santos et al., 2014). Se recolectaron cuatro ejemplares por número de colección, para la respectiva exsiccata botánica y muestras de los órganos vegetales, para la réplica de extracción del tinte. Las especies fueron identificadas mediante el uso de claves taxonómicas, bibliografía especializada según las familias botánicas reportadas (Brako \& Zarucchi, 1993; Gentry, 1993; Reynel et al., 2007; Weberbauer, 1945), herbarios virtuales como Neotropical Herbarium, Plants Jstor, Kew Herbarium y comparaciones con material de los herbarios USM y MOL. La clasificación taxonómica se basó en el sistema de clasificación APG IV (APG IV, 2016). Los ejemplares botánicos, debidamente clasificados y herborizados, fueron depositados en el Herbario USM de la Universidad Nacional Mayor de San Marcos y colección HEPLAME de la Universidad Peruana Cayetano Heredia.

Registro de la información etnobotánica.

En la búsqueda de la reconstrucción de técnicas tintóreas textiles en base a plantas, se aplicó la caminata etnobotánica, la cual consistió en el recorrido por los alrededores de las comunidades visitadas, con la participación de los miembros de la comunidad. En el caso de las comunidades de Pamparomas (Ancash) y Pampaphalla (Cusco), se contó con la participación del grupo de mujeres tintoreras del distrito, que forman parte de proyectos auspiciados por ONGs regionales.

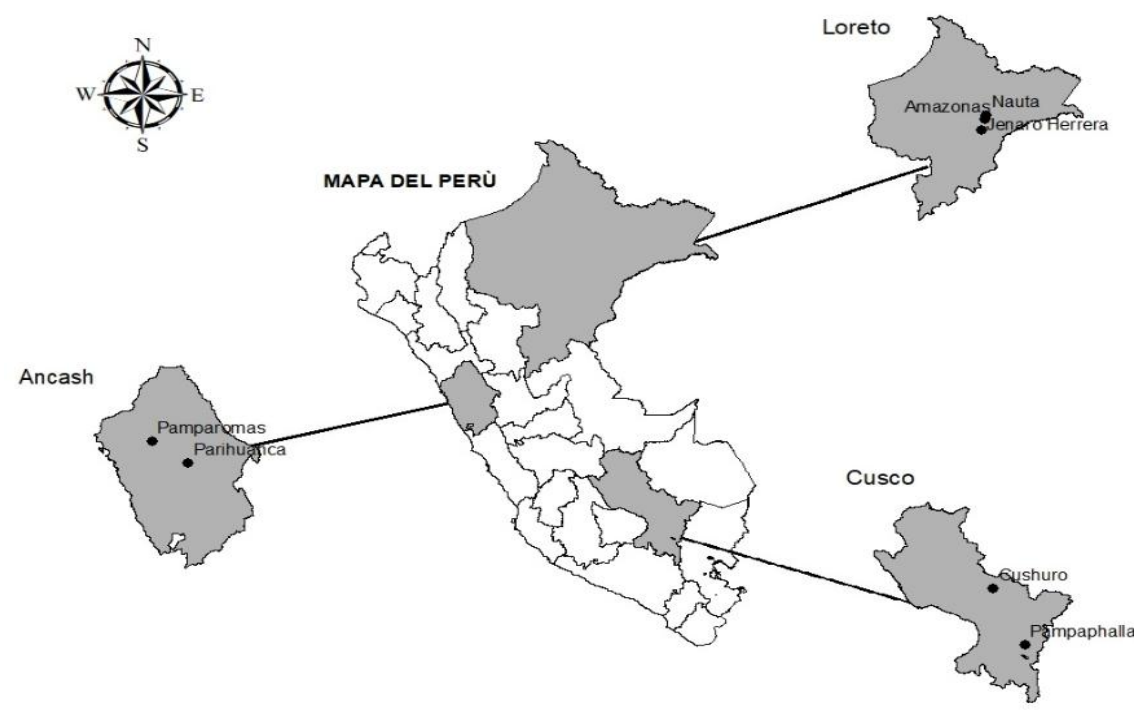

Figura 1. Mapa de localización de las comunidades estudiadas en los departamentos de Loreto, Ancash y Cusco. 
Tabla 1. Localidades de estudio y nombres de los participantes en las Comunidades /caserío.

\begin{tabular}{|c|c|c|c|c|c|c|}
\hline $\begin{array}{l}\text { Comunidad } \\
\text { / Caserío } \\
\end{array}$ & Distrito & Provincia & Región & Coordenadas & $\begin{array}{l}\text { Altitud } \\
\text { (msnm) }\end{array}$ & $\begin{array}{l}\text { Nombre del } \\
\text { poblador local }\end{array}$ \\
\hline Jenaro & Jenaro & Requena & Loreto & ${\mathrm{S} 04^{\circ} 54^{\prime} 10.8^{\prime \prime}}$ & 50 & Dora Tangoa, Elia \\
\hline Herrera & Herrera & & & $\mathrm{O} 73^{\circ} 39^{\prime} 58.4^{\prime \prime}$ & & $\begin{array}{l}\text { Canayo, Doilith } \\
\text { del Castillo }\end{array}$ \\
\hline Amazonas & Nauta & Nauta & Loreto & $\begin{array}{l}\mathrm{S} 04^{\circ} 27^{\prime} 34.9^{\prime \prime} \\
\mathrm{O} 73^{\circ} 31^{\prime} 32.9^{\prime \prime}\end{array}$ & 55 & Francisca Orosco \\
\hline $\begin{array}{l}\text { Carretera } \\
\text { Iquitos- } \\
\text { Nauta km } \\
60.2\end{array}$ & Iquitos & Maynas & Loreto & $\begin{array}{l}\mathrm{S} 04^{\circ} 16^{\prime} 06.5^{\prime \prime} \\
\mathrm{O} 73^{\circ} 29^{\prime} 29.0^{\prime \prime}\end{array}$ & 45 & Julia Ruiz Miteco \\
\hline Pariahuanca & $\begin{array}{l}\text { San Miguel } \\
\text { de Aco }\end{array}$ & Carhuaz & Ancash & $\begin{array}{l}\mathrm{S} 09^{\circ} 22^{\prime} 9.01^{\prime \prime} \\
\mathrm{O} 77^{\circ} 33^{\prime} 47.88^{\prime \prime}\end{array}$ & 2800 & $\begin{array}{l}\text { Olimpio Castillo, } \\
\text { Antonio Aquitari }\end{array}$ \\
\hline Pamparomas & Pamparomas & Huaylas & Ancash & $\begin{array}{l}\mathrm{S} 09^{\circ} 4^{\prime} 12.58^{\prime \prime} \\
\mathrm{O} 77^{\circ} 58^{\prime} 45.48^{\prime \prime}\end{array}$ & 2650 & $\begin{array}{l}\text { America Tamaris, } \\
\text { Triscila Gonzales, } \\
\text { Dora Rivera, } \\
\text { Octavia Escalante, } \\
\text { Octavia Ramos }\end{array}$ \\
\hline Pampaphalla & Sicuani & Canchis & Cusco & $\begin{array}{l}\mathrm{S} 14^{\circ} 14^{\prime} 09.8^{\prime \prime} \\
\mathrm{O} 71^{\circ} 12^{\prime} 12.1^{\prime \prime}\end{array}$ & 3300 & Laura Caballero \\
\hline Cushuro & Challabamba & Paucartambo & Cusco & $\begin{array}{l}\mathrm{S} 13^{\circ} 01^{\prime} 46.4^{\prime \prime} \\
\mathrm{O} 71^{\circ} 47^{\prime} 22.1^{\prime \prime}\end{array}$ & 2850 & $\begin{array}{l}\text { Josefina Llacta, } \\
\text { Savino Cárdenas, } \\
\text { Jaime Cárdenas }\end{array}$ \\
\hline
\end{tabular}

Se aplicaron entrevistas semiestructuradas en la recolección de la información etnobotánica (Albán, 1985; Albuquerque et al., 2014) (Anexo 1). Las comunidades ubicadas en la región Loreto, correspondieron a poblaciones mestizas, descendientes Cocamas, ubicadas en áreas rurales con acceso a servicios básicos. Las comunidades ubicadas en las regiones de Cusco y Ancash corresponden a poblaciones mestizas, descendientes Quechua, ubicadas en áreas urbanas con acceso a los servicios básicos.

Se aplicó el método de simulación (Albuquerque et al. 2014) para la reconstrucción de las técnicas tradicionales, para lo cual se utilizaron fibras de origen animal (lana de oveja y alpaca) y vegetal (Astrocaryum chambira Burret), y los respectivos mordientes y fijadores, según las indicaciones registradas en las entrevistas. Se realizaron 16 entrevistas solicitándose el consentimiento informado a los pobladores participantes en el estudio (Tabla 1).

\section{Resultados.}

En el ámbito andino, las fibras textiles usadas son preferentemente de origen animal (oveja, alpaca), mientras que, en la Amazonía, son utilizados con mayor frecuencia las fibras vegetales extraídas a partir de las hojas de especies de palmeras (nervaduras). Como parte del proceso de replicación de las técnicas empleadas en las comunidades de estudio, se observó que el proceso de teñido consta de cuatro fases fundamentales, los cuales se dan de manera consecutiva: a) Penetración y difusión del colorante disuelto en agua en la superficie de la fibra, b) Adhesión del colorante a la superficie de la fibra, c) Difusión del colorante de la capa superficial al interior de la fibra y d) Fijación del colorante en el interior de la fibra. Como paso previo a la obtención del tinte, la preparación de las fibras resulta ser fundamental, implicando la eliminación de las impurezas en la fibra textil, dado que estos, no solo interrumpen la unión del tinte al material a teñir, sino que también dificulta el buen acabado del producto. Dependiendo de la naturaleza de la fibra utilizada, se observaron diferencias en cuanto al tratamiento de ésta según se procese fibras de origen animal o vegetal.

Adhesión del colorante (uso de mordientes).

Dependiendo del momento en el cual se aplica, se denomina pre-mordentado, cuando la lana se somete al mordiente antes de la tinción, y post-mordentado, cuando este se ejecuta luego de realizarse el teñido. El pre-mordentado facilita la captación y fijación de los colorantes disueltos. Por ejemplo, para 500 gramos de lana de oveja se requiere aproximadamente $50 \mathrm{~g}$ de alumbre $\left(\mathrm{KAl}\left(\mathrm{SO}_{4}\right)_{2} \bullet 12 \mathrm{H}_{2} \mathrm{O}\right)$ previamente triturada; luego, se vierte en la olla con agua caliente la lana de oveja lavada en el alumbre y se deja hervir por 30 minutos; finalmente se escurre y se lava la lana con abundante agua. Se deja reposar la lana hasta el momento del teñido. El Post-mordentado tiene como objetivo variar el color adquirido durante la tinción y reforzar la solidez al lavado y a la exposición a la luz (Cedano, 1994).

Tinción.

Esta etapa consiste en la obtención de la materia prima vegetal (tallos, hojas, semillas, frutos) los cuales son sometidos a trituración o machacado con el fin de romper tejidos vegetales y liberar los pigmentos localizados en el citoplasma de la célula. El tiempo de reposo (remojo) varía según la especie utilizada, pudiendo ser este desde horas a días. Luego de mezclar 

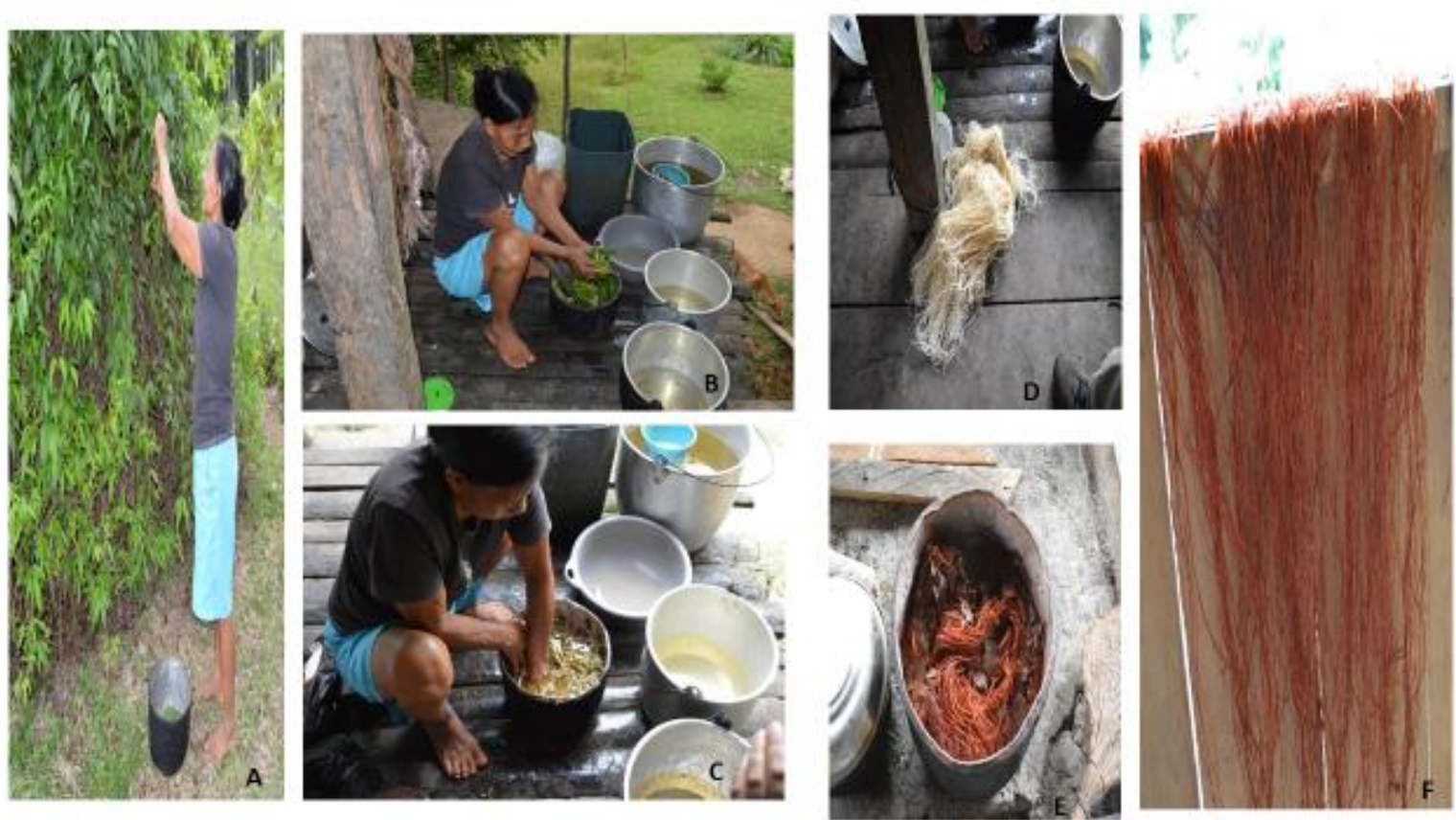

Figura 2. A. Señora Julia Ruiz Mibeco (comunidad Bora de Brionuevo, río Ampiyacu, Pebas), recolectando hojas de "pucapanga" (Fridericia chica (Bonpl.) L.G. Lohmann) para la tinción de fibras de "chambira" (Astrocaryum chambira Burret) B. Proceso de rallado de las hojas de "pucapanga". C. Fibra de "chambira". D. Mezcla de la fibra de "chambira" junto con las hojas ralladas de "pucapanga" y agua. E. Adhesión del tinte rojo obtenido de "pucapanga" a la chambira luego de la decocción, 30 minutos. F. Secado de la fibra ya teñida.

la materia prima a usar, en agua junto con la madeja de la fibra a teñir; se procede a la cocción a fuego lento durante 30-60 minutos aproximadamente, dependiendo de la intensidad de color que se desee obtener. En el caso del uso de semillas se procede a removerlas o frotar las semillas para extraer la testa pulposa. Ejemplo de ello fue el caso del uso de las semillas del Bixa orellana L.

Penetración del colorante en fibras de origen animal (lana de oveja o alpaca).

La fibra animal debe ser lavada con detergente o jabón antes de ser sometida a la tinción. Ello permite eliminar la grasa o residuos de suciedad existente en este. Se debe evitar frotar bruscamente la fibra, dado que podría enredarse o deshilacharse. Lo adecuado es moverla suavemente en círculos dentro del recipiente, repitiendo el proceso cuantas veces sea necesario.

Penetración del colorante en fibras de origen vegetal: (Hojas de Astrocaryum chambira Burret).

Las fibras de Astrocaryum chambira Burret se colocan en un recipiente y se procede a hervirlas con abundante jugo de "toronja" (Citrus paradisi Macfad.). Luego, se retira la fibra y se enjuaga con abundante agua. Se escurre y se deja secar al Sol (Figura 2).

Enjuague y secado.

Con el propósito de eliminar el exceso de tinte en la fibra se procede a enjuagar en agua, hasta que el agua obtenida sea transparente. Finalmente, se deja secar la fibra bajo sombra. El uso de las raíces de Colletia spinossisima J. F. Gmel., en el lavado de la lana, le brinda brillo y suavidad (Cornejo, 1987).

Plantas utilizadas en la tinción de las fibras.

De la gama de colores obtenidos, de acuerdo con el modelo Red-Green-Blue Color Space (RGB), (Pascale, 2003), los colores primarios rojo, verde y azul se obtuvieron en un $52.93 \%$ de las especies registradas. Mientras que, en la gama de colores secundarios, el amarillo, se obtuvo en un $13.72 \%$. Como color terciario, el marrón, se obtuvo en el $21.56 \%$ de las especies con las que se experimentó (Tabla 2). Finalmente, los colores obtenidos de las fibras fueron codificados atendiendo la tabla Munsell Color (Munsell, 1912), a fin de estandarizar especificaciones en torno al color obtenido y minimizar subjetividades.

Se registran 51 especies de uso tintóreo para el ámbito de estudio, incluidas en 45 géneros y 30 familias botánicas. La familia Asteraceae registra el mayor número de incidencias de usos como tintóreo, seguida por la familia Fabaceae (Figura 3). En el Anexo 2 se indican las especies utilizadas en la actualidad en el proceso de tinción con el detalle del órgano de la planta empleado y tipo de fibra utilizada.

El órgano de la planta con mayor registro de uso fueron las hojas $(57 \%)$, seguido por los tallos (24\%) (Figura 4). 
Tabla 2. Porcentaje de especies según el color obtenido.

\begin{tabular}{lcc}
\hline COLOR & NUMERO ESPECIES & \% \\
\hline verde & 17 & 33.33 \\
marrón & 11 & 21.56 \\
rojo & 9 & 17.64 \\
amarillo & 7 & 13.72 \\
morado & 2 & 3.92 \\
plomo & 2 & 3.92 \\
abanó & 1 & 1.96 \\
azul & 1 & 1.96 \\
granate & 1 & 1.96 \\
guinda & 1 & 1.96 \\
negro & 1 & 1.96 \\
purpura & 1 & 1.96 \\
violeta & 1 & 1.96 \\
anaranjado & 1 & 1.96 \\
rosado & 1 & 1.96 \\
\hline
\end{tabular}

\section{Discusión.}

En el ámbito andino y amazónico, la actividad tintórea en textiles es realizada especialmente por las mujeres, poseedoras del conocimiento tradicional y ejes importantes en el proceso de la transmisión (Junsongduang et al., 2017; Paván et al., 2016; Rojas et al., 2016).

Algunos tintes vegetales requieren del uso de mordientes (sustancias químicas, sales metálicas solubles en agua, que facilitan la unión entre el tinte y la fibra) para su fijación en la fibra. El color obtenido, no solo dependerá de la fuente, sino también del mordiente y el elemento a teñir (Boucherie, 2014). Pobladores de las comunidades andinas de las regiones de Ancash y Cusco utilizan, hasta la actualidad, mordientes de origen mineral, siendo el alumbre (Sulfato doble de aluminio y potasio), el más ampliamente utilizado en los Andes; mientras que en la región de Loreto (Nauta, Maynas y Requena), solo se precisó el uso del zumo de algún cítrico ("toronja") como medio para matizar los colores y fijarlo. El uso del patsu, corpa o caparrosa (sulfato de cobre) y orina humana, fue registrado solo en comunidades de la provincia de Huaylas. En el proceso de tinción, Cornejo
(1987) recomienda que, en el caso del uso de las raíces, estas deben ser secadas y molidas antes de someterlas a la cocción. En el caso del uso de frutos, estos deben ser secados y molidos, salvo excepciones como en Caesalpinia spinosa (Molina) Kuntze, en donde las semillas deben ser separadas de las vainas con anterioridad a la cocción; o de Solanum nitidum Ruiz \& Pav., en el cual estos deben ser fermentados previamente antes de la cocción. En el proceso de lavado, se reporta el uso de especies como Colletia spinosissima J.F. Gmel., Solanum hispidum Pers. y Agave americana L., durante el proceso del lavado (Cornejo, 1987).

De las 51 especies registradas en las siete localidades estudiadas, seis especies (Baccharis genistelloides (Lamarck) Persoon, Bidens pilosa L., Juglans neotropica Diels, Mutisia acuminata Ruíz \& Pav., Baccharis latifolia (Ruíz \& Pav.) Pers., y Buddleja coriacea Remy) comparten el conocimiento de uso en comunidades de las regiones de Cusco y Ancash. La especie andina conocida como "mullaca" (Muehlenbeckia volcanica (Benth) Endl.), probablemente es la especie que muestra mayor resiliencia en el conocimiento de uso, mencionada desde 1653, en la obra "Historia del nuevo mundo" de Bernabé Cobo. De los frutos, raíces y hojas de esta especie se obtuvieron los colores azul, morado y negro (Contreras, 2009), y de la planta entera, el color marrón. En relación con el "añil", Indigofera suffruticosa Mill., para la obtención del color azul, Arriaga et al. (2013) refieren la presencia de un diterpeno lineal, ésteres de ácidos grasos y compuestos alifáticos en esta especie. Este diterpeno se encuentra en la planta en forma de Indicán que por hidrólisis (por fermentación o con ácidos) se descompone en glucosa e indoxil. Este último se oxida al aire inmediatamente y queda transformado en indigotina (Quintanilla, 2005). Sin embargo, el rendimiento y composición química depende del estadio de crecimiento, parte de la planta a utilizar, secado poscosecha y temperatura (Silva, 2014). Ensayos en hojas secas fueron realizados por Ushida (2004), en la que menciona que la remoción

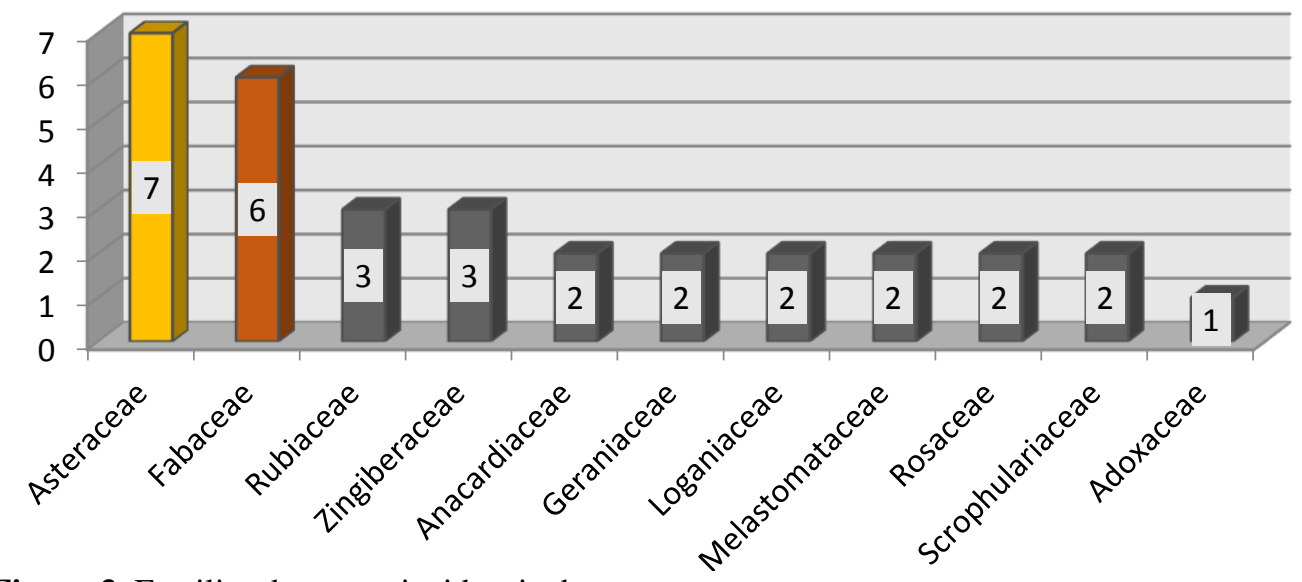

Figura 3. Familias de mayor incidencia de usos. 
rápida de la humedad en las hojas frescas produce Indicán. Sin embargo, si las hojas frescas se secan lentamente, la hidrólisis del Indicán (para formar indigotina) puede llevarse a cabo por la enzima de Indigofera cuya actividad es lenta. Esto explica por qué al utilizar la técnica estandarizada de tinción en Indigofera, la cual incluye el secado previo de la planta, no se haya obtenido el color azul esperado, debido al bajo porcentaje de Indicán presente, obteniéndose el color marrón oscuro.

En relación con el "huito", Genipa americana, su uso como tinte para fibra es bastante generalizado, obteniéndose los colores negro y azul, dependiendo de la cantidad de fruto a utilizar. Del "huito" se utiliza la pulpa del fruto, la cual es picada y cocinada junto con las fibras de "chambira". Sin embargo, en la comunidad Amazonas, las artesanas de la asociación artesanal El Palo Sangre, prefieren utilizar las semillas, dado que fija mejor el color. En relación con el tinte rojo, cuyo color se obtuvo en el $17.64 \%$ de las especies registradas, la planta conocida como "pampa chapi" Galium hypocarpium (L.) Endl. ex Griseb., fue encontrada en Cusco. Sin embargo, los pobladores mencionaron que no es muy usada, debido a la dificultad para obtener la raíz, la misma que, siendo pivotante, delgada y de pequeña longitud, requeriría ser recolectada en grandes extensiones. Esta situación no favorece el uso de $G$. hypocarpium, dado que no es abundante en la zona. Ante ello, los pobladores prefieren utilizar otra especie para la obtención de tinte rojo, la Calceolaria leptantha Pennell, popularmente conocida como "chapi rojo". C. leptantha es un arbusto relativamente abundante en el matorral húmedo de la región Cusco, lo cual permite un fácil acceso a cantidades necesarias de materia prima para realizar actividades de tinción.

\section{Conclusiones.}

El uso de plantas tintóreas en comunidades andinas y amazónicas peruanas es de amplio conocimiento e inherente a su cultura; sin embargo, en la actualidad su uso ha sido observado solo a nivel de las mujeres artesanas, para propósitos de venta, como parte de su economía familiar. Su identidad cultural es reflejada a través de la conservación de las técnicas empleadas para la obtención del tinte y las formas de fijación de este sobre los textiles y objetos artesanales.

El estudio registra 51 especies de la flora nativa peruana asociada al conocimiento tradicional, reconociéndose que dicha propiedad intelectual es exclusiva para cada una de las comunidades mencionados en el artículo. Los colores obtenidos con mayor frecuencia fueron el verde (primario), amarillo (secundario) y el marrón (terciario), y de preferencia las plantas deben ser utilizadas frescas.

\section{Agradecimientos.}

El estudio fue realizado bajo los auspicios de los proyectos FINCYT-Innóvate Perú (contrato $\mathrm{N}^{\circ} 130$ FINCyT -IA- 2013), y FEDU No 141001211. Los autores agradecen a cada uno de los pobladores de las regiones de Ancash (Pamparomas, San Miguel de Aco), Cusco (Sicuani, Challabamba) y Loreto (Iquitos, Nauta, Jenaro Herrera) por compartir con nosotros su sabiduría y conocimientos vivos aun en su memoria. Estudios posteriores que se realicen en base a los resultados obtenidos deberán involucrar y reconocer los derechos de los tintoreros tradicionales, de acuerdo con las normas legales vigentes en el Perú.

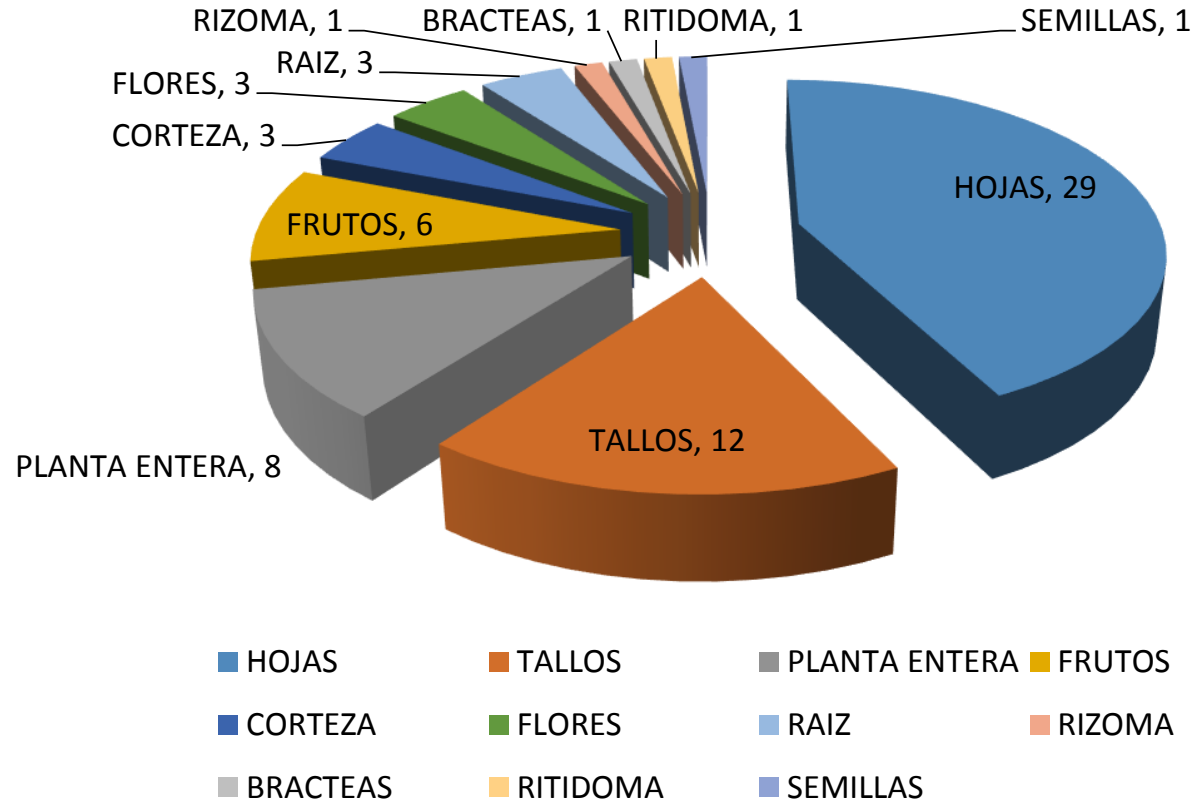

Figura 4. Órgano de la planta utilizado como tintóreo. 
Literatura citada.

Albán J. 1985. Un registro de datos etnobotánicas. Boletín de Lima 7 (39): 93-96.

Albuquerque U.P., Cruz da Cunha L.V.F., Lucena R.F.P. \& Alves, R.R.N. (Editors). 2014. Methods and Techniques in Ethnobiology and Ethnoecology Humana Press. 476 p.

Antúnez de Mayolo K. 1989. Peruvian Natural Dye Plants. Economic Botany 43 (2): 181-191.

APG IV (Angiosperm Phylogeny Group IV). 2016. An update of the Angiosperm Phylogeny Group classification for the orders and families of flowering plants. APG IV. Botanical Journal of the Linnean Society 181 (1): 1-20.

Arriaga A.M., Lemos T.L, Santiago G.M., Andrade-Neto M., Braga M.A., De Almeida M.C., Gomes T.B.M., Rodrigues F.E.A., Nunes e Vasconcelos J. \& Alves P.B. 2013. Chemical composition and antioxidant activity of Indigofera suffruticosa. Chemistry of Natural Compounds 49 (1): 150-151.

Boucherie N. 2014. La couleur dans la civilisation nasca: production tinctoriale et picturale, Tesis De Doctorado. Lyon, Université Lumière Lyon 2.

Brack A. 1999. Diccionario Enciclopédico de plantas útiles del Perú. Cusco. Perú: PNUD / CBC, 566 p., 24 láminas.

Brako L. \& Zarucchi, J. 1993. Catalogue of the flowering plants and gymnosperms of Peru. Missouri Botanical Gardens, St. Louis, Mo.

Cedano M. 1994. Teñido con Hongos. Folleto instructivo, Universidad de Guadalajara, Instituto de Botánica, Guadalajara.

Cerrate E. 1969. Manera De Preparar Plantas Para Un Herbario. UNMSM. Museo De Historia Natural., serie de divulgación 1.

Contreras V. 2009. Textiles y tintes naturales en Quico, una comunidad Q'ero. Primera edición. Cusco, Perú.

Cornejo Z. M. 1987 Características textiles del contexto de enterramiento La Horca Camaná. Arequipa: Universidad Católica Santa María. Tesis de maestría.

Gentry A. 1993. Field Guide to the Families and Genera of Woody Plants of Northwest South America (Colombia, Ecuador, Peru) with supplementary notes on herbaceous taxa. Washington, DC. $895 \mathrm{p}$.

Hurtado Díaz M. \& Rojas J. M. 2005. El color que no se olvida: Tintes naturales en la tradición campesina de Cajamarca. Red de Bibliotecas Rurales de Cajamarca.

Ivic de Monterroso M. \& Berger M. 2008. El arte sale de las casas. Los tintes naturales en San Juan la Laguna Solola. Ciencia y Técnica Maya. Fundación Solar. Guatemala.

Jansen P.C.M. \& Cardon, D. (Editors). 2005. Plant resources of Tropical Africa 3. Dyes and tannins. PROTA Foundation, Wageningen, Netherlands/Backhuys publishers, Leiden, Netherlands/CTA, Wageningen, Netherlands. 216 p.

Junsongduang A., Sirithip K., Inta A., Nachai R., Onputtha B, Tanming W. \& Balslev H. 2017. Diversity and Traditional Knowledge of Textile Dyeing Plants in northeastern Thailand. Economic Botany, 71 (3): 241255.

Lima dos Santos L., Vieira F., Gomes de Sousa L., Oliveira da Silva A., Lima dos Santos L. \& de Sousa G.M. 2014. Techniques for Collecting and Processing Plant Material and Their Application in Ethnobotany Research. pp 161173. In: Methods and Techniques in Ethnobiology and Ethnoecology. U. Albuquerque, L. Vital Fernandes C. da Cunha, R. Farias Paiva de Lucena, R. Nobrega Alves. Editors. 2014. Humana Press. 476 p.

Munsell A. H. 1912. A pigment color system and notation. The American Journal of Psychology. University of Illinois Press. 23 (2): 236-244.

Pascale D. 2003. A review of RGB Color Spaces. The Babel Color Company. Canada.

Paván M., Furlan V., Renny M., Monterroso I. \& Argüello L. 2017. Tintes naturales vegetales en el paraje el desmonte, reserva cultural-natural Cerro Colorado, Córdoba (Argentina). BONPLANDIA 26 (2): 103-113.

Poma B. 2016. Análisis de la situación actual de teñido natural en la provincia de Loja. Tesis. Universidad de Azuay. Facultad de Diseño. Cuenca, Ecuador.

Quintanilla R.E. 2005. Guía técnica: Procesamiento del Añil en El Salvador. OEA, IICA, GTZ, Santa Tecla, El Salvador.

Reynel C., Pennington R., Pennington J., Marcelo J. \& Daza A. 2007. Arboles útiles del Ande peruano y sus usos: un manual con apuntes de identificación, ecología y propagación de las especies de la Sierra y los Bosques Montanos en el Perú. Herbario de la Facultad de Ciencias Forestales de la Universidad Nacional Agraria-La Molina, Royal Botanic Gardens Kew, Royal Botanic Gardens Edinburgh, APRODES.

Rojas R., Díaz C., Espinoza G., Figari J. \& Albán J. 2016. Plantas Tintóreas Peruanas. Universidad Peruana Cayetano Heredia.

Silva C.B. 2014. Avaliação do potencial biológico de óleos essenciais e extratos orgânicos de folhas de Indigofera suffruticosa. Tese de Doutorado apresentada ao programa de Pós-Graduação em Bioquímica e Fisiologia da Universidade Federal de Pernambuco.

Sing de Ugaz, O. L. 1997. Colorantes naturales. Fondo Editorial, Pontificia Universidad Católica de Perú. 274 p.

Trilla C., Demaio P., Colantonio S. \& Galetto L. 2007. Conocimiento actual de plantas tintóreas por los pobladores del valle de Guasapampa, provincia de Córdoba. KURTZIANA. Volumen especial de Etnobotánica. 33 (1): 65-71.

Ushida S. 2004. Extracción de añil con hojas secas. Primer Congreso Internacional del Añil y otros colorantes. San Salvador, El Salvador.

Weberbauer A. 1945. El Mundo Vegetal de los Andes Peruanos, Estudio Fitogeográfico. Ministerio de Agricultura, Dirección de Agricultura, Estación Experimental agrícola de La Molina, Lima. 776 p.

Zumbuhl H. 1979 Tintes naturales para lana de oveja. Karmaq maki, Sepas, Huancayo. 90 p. 
Anexo 1.

$$
\begin{aligned}
& \mathrm{N}^{\circ} \mathrm{de} \\
& \text { encuesta }
\end{aligned}
$$

NOMBRE DEL INFORMANTE

EDAD

LUGAR DE NACIMIENTO

NOMBRE COMÚN DE LA PLANTA

FECHA DE LA ENTREVISTA

1. Qué color obtiene

2. Qué tipo de lana utiliza

3. Cuanto usa de la planta $(\mathrm{kg}) \mathrm{Vs}$ cantidad de lana a teñir

4. Utiliza mordientes, cuáles, ¿cuánto?

6. ¿QUÉ PARTE(S) DE LA PLANTA EMPLEA?

a) Raíz b) Tallo c) Hojas d) Flores e) Frutos

f) Semillas g) Corteza h) Planta entera i) Otras...

7. Explique el procedimiento a seguir en la tinción.
Ficha etnobotánica según Albán (1985)

Para la recopilación de la información sobre plantas tintóreas

Reconstrucción de las Técnicas tintóreas Precolombinas, en base a Productos Naturales, para su preservación, revalorización y uso en la artesanía e industria textil contemporánea

Anexo 2. Especies tintóreas usadas en comunidades andinas y amazónicas peruanas.

\begin{tabular}{|c|c|c|c|c|c|c|c|}
\hline Familia/ Género/ Especie & $\begin{array}{l}\text { Nombre } \\
\text { vernáculo }\end{array}$ & $\begin{array}{l}\text { Órgano } \\
\text { empleado }\end{array}$ & $\begin{array}{l}\text { Color } \\
\text { obtenido }\end{array}$ & $\begin{array}{l}\text { Código } \\
\text { Munsell }\end{array}$ & $\begin{array}{l}\text { Tipo de } \\
\text { fibra } \\
\text { utilizada }\end{array}$ & $\begin{array}{l}\text { Región / } \\
\text { Comunidad } \\
\text { que lo } \\
\text { aplica } \\
\text { Localidad } \\
\end{array}$ & Exsiccatae \\
\hline $\begin{array}{l}\text { ADOXACEAE } \\
\text { Sambucus peruviana } \\
\text { Kunth }\end{array}$ & Rayan & Hojas & Verde claro & $5 \mathrm{GY} 6 / 4$ & $\begin{array}{ll}\text { Lana } & \text { de } \\
\text { oveja }\end{array}$ & $\begin{array}{l}\text { Ancash, } \\
\text { Quebrada de } \\
\text { Sokup, } \\
\text { Huaylas }\end{array}$ & $\begin{array}{l}\text { JAC-6674 } \\
\text { CD-13107 }\end{array}$ \\
\hline $\begin{array}{l}\text { ANACARDIACEAE } \\
\text { Schinus molle L. }\end{array}$ & Molle & Hojas & Verde & $10 \mathrm{Y} 3 / 4$ & $\begin{array}{l}\text { Lana de } \\
\text { oveja }\end{array}$ & $\begin{array}{l}\text { Cusco, } \\
\text { Pampamaca, } \\
\text { San Miguel } \\
\text { de Aco }\end{array}$ & CD-13095 \\
\hline $\begin{array}{l}\text { Tapirira guianensis } \\
\text { Aublet. }\end{array}$ & Huaira caspi & $\begin{array}{l}\text { Frutos, } \\
\text { hojas }\end{array}$ & Marrón & --- & Chambira & $\begin{array}{l}\text { Loreto, } \\
\text { SantoTomás } \\
\text { - Río Nanay, } \\
\text { Loreto }\end{array}$ & CD-13145 \\
\hline $\begin{array}{l}\text { ANNONACEAE } \\
\text { Guatteria flabellata Erk } \\
\text { ens \& Maas. }\end{array}$ & Sin referencia & Hojas & Granate & --- & Chambira & $\begin{array}{l}\text { Loreto, } \\
\text { Santo Tomás } \\
\text { - Río Nanay, } \\
\text { Loreto }\end{array}$ & CD-13112 \\
\hline $\begin{array}{l}\text { ARECACEAE } \\
\quad \text { Euterpe precatoria Mart. }\end{array}$ & Huasaí & Frutos & Guinda & --- & Chambira & $\begin{array}{l}\text { Loreto, } \\
\text { Santo Tomás } \\
\text { - Río Nanay, } \\
\text { Loreto }\end{array}$ & CD-13119 \\
\hline $\begin{array}{l}\text { ASTERACEAE } \\
\text { Aristeguietia discolor } \\
\text { (DC) King \& H. Rob. }\end{array}$ & Quillua & Ramas & Verde & & $\begin{array}{l}\text { Lana de } \\
\text { oveja }\end{array}$ & $\begin{array}{l}\text { Ancash, } \\
\text { Quipia, } \\
\text { Huaylas }\end{array}$ & $\begin{array}{l}\text { JAC-8394 } \\
\text { CD-13104 }\end{array}$ \\
\hline $\begin{array}{l}\text { Baccharis genistelloides } \\
\text { (Lamarck) Persoon }\end{array}$ & Kimsa cuchu & $\begin{array}{l}\text { Planta } \\
\text { entera }\end{array}$ & Verde & $5 \mathrm{G} 1 / 2$ & $\begin{array}{l}\text { Lana de } \\
\text { oveja }\end{array}$ & $\begin{array}{l}\text { Cusco, } \\
\text { Pampamaca, } \\
\text { San Miguel } \\
\text { de Aco }\end{array}$ & CD-13097 \\
\hline
\end{tabular}


Anexo 2. Especies tintóreas usadas en comunidades andinas y amazónicas peruanas.

\begin{tabular}{|c|c|c|c|c|c|c|c|}
\hline Familia/ Género/ Especie & $\begin{array}{l}\text { Nombre } \\
\text { vernáculo }\end{array}$ & $\begin{array}{l}\text { Órgano } \\
\text { empleado }\end{array}$ & $\begin{array}{l}\text { Color } \\
\text { obtenido }\end{array}$ & $\begin{array}{l}\text { Código } \\
\text { Munsell }\end{array}$ & $\begin{array}{l}\text { Tipo de } \\
\text { fibra } \\
\text { utilizada }\end{array}$ & $\begin{array}{l}\text { Región / } \\
\text { Comunidad } \\
\text { que lo } \\
\text { aplica / } \\
\text { Localidad } \\
\end{array}$ & Exsiccatae \\
\hline $\begin{array}{l}\text { Baccharis latifolia (R. \& } \\
\text { P.) Persoon }\end{array}$ & Chilca & Hojas & Verde & $2.5 \mathrm{GY} 2 / 4$ & $\begin{array}{ll}\text { Lana de } \\
\text { alpaca }\end{array}$ & $\begin{array}{l}\text { Cusco, } \\
\text { Pampamaca, } \\
\text { San Miguel } \\
\text { de Aco, } \\
\text { Pampaphalla } \\
\text {, Sicuani }\end{array}$ & $\begin{array}{l}\text { CD-13100, } \\
\text { JAC-19184 }\end{array}$ \\
\hline Bidens pilosa $\mathrm{L}$. & Amor seco & $\begin{array}{l}\text { Planta } \\
\text { entera }\end{array}$ & Amarillo & & $\begin{array}{l}\text { Lana de } \\
\text { oveja }\end{array}$ & $\begin{array}{l}\text { Ancash, } \\
\text { Quebrada de } \\
\text { Sokup, } \\
\text { Huaylas }\end{array}$ & JAC-6833 \\
\hline $\begin{array}{l}\text { Mutisia acuminata Ruiz } \\
\text { \& Pav. }\end{array}$ & Chinchircuma & Flores & Amarillo & & $\begin{array}{l}\text { Lana de } \\
\text { alpaca }\end{array}$ & $\begin{array}{l}\text { Cusco, } \\
\text { Pampaphalla } \\
\text {, Sicuani }\end{array}$ & JAC-7262 \\
\hline $\begin{array}{l}\text { Ophryosporus chilca } \\
\text { (Kunth) Hieron }\end{array}$ & Shequia & $\begin{array}{l}\text { Planta } \\
\text { entera }\end{array}$ & $\begin{array}{l}\text { Verde, } \\
\text { amarillo }\end{array}$ & $5 \mathrm{GY} 3 / 4$ & $\begin{array}{l}\text { Lana de } \\
\text { alpaca }\end{array}$ & $\begin{array}{l}\text { Cusco, } \\
\text { Pampamaca, } \\
\text { San Miguel } \\
\text { de Aco }\end{array}$ & CD-13101 \\
\hline $\begin{array}{l}\text { Ophryosporus } \\
\text { peruvianus R.M. King \& } \\
\text { H. Rob. }\end{array}$ & Sayanco & Ramas & Verde & & $\begin{array}{l}\text { Lana de } \\
\text { oveja }\end{array}$ & $\begin{array}{l}\text { Ancash, } \\
\text { Quebrada de } \\
\text { Sokup, } \\
\text { Huaylas }\end{array}$ & JAC-19193 \\
\hline $\begin{array}{l}\text { BETULACEAE } \\
\quad \text { Alnus acuminata Kunth }\end{array}$ & Aliso & Hojas & $\begin{array}{l}\text { Marrón } \\
\text { oscuro }\end{array}$ & $10 \mathrm{Y} 1 / 2$ & $\begin{array}{l}\text { Lana de } \\
\text { oveja }\end{array}$ & $\begin{array}{l}\text { Ancash, } \\
\text { Quebrada de } \\
\text { Sokup, } \\
\text { Huaylas }\end{array}$ & $\begin{array}{l}\text { JAC-5621 } \\
\text { CD-13110 }\end{array}$ \\
\hline $\begin{array}{l}\text { BIGNONIACEAE } \\
\text { Fridericia chica } \\
\text { (Bonpl.) L.G.Lohmann }\end{array}$ & Pucapanga & Hojas & Rojo & 10R3/10 & Chambira & $\begin{array}{l}\text { Loreto, } \\
\text { Carretera } \\
\text { Iquitos- } \\
\text { Nauta km } \\
60.2\end{array}$ & CD-13120 \\
\hline $\begin{array}{l}\text { BIXACEAE } \\
\quad \text { Bixa orellana } \mathrm{L} .\end{array}$ & Achote, achiote & Semillas & $\begin{array}{l}\text { Anaranjado } \\
\text { Rojo }\end{array}$ & $\begin{array}{l}2.5 \mathrm{YR} 6 / 14 \\
10 \mathrm{R} 3 / 8\end{array}$ & Chambira & $\begin{array}{l}\text { Loreto, } \\
\text { Jenaro } \\
\text { Herrera, } \\
\text { Loreto; } \\
\text { Pampaphalla } \\
\text {, Sicuani }\end{array}$ & CD-13124 \\
\hline $\begin{array}{l}\text { BRASSICACEAE } \\
\text { Nasturtium officinale } \\
\text { R.Br. }\end{array}$ & Berros & $\begin{array}{l}\text { Planta } \\
\text { entera }\end{array}$ & Verde & $5 \mathrm{GY} 5 / 6$ & $\begin{array}{l}\text { Lana de } \\
\text { alpaca }\end{array}$ & $\begin{array}{l}\text { Cusco, } \\
\text { Pampamaca, } \\
\text { San Miguel } \\
\text { de Aco }\end{array}$ & CD-13098 \\
\hline $\begin{array}{l}\text { BROMELIACEAE } \\
\text { Tillandsia usneoides (L.) } \\
\text { L. }\end{array}$ & $\begin{array}{l}\text { Salvajina, } \\
\text { Kacasunka, } \\
\text { barba del cerro }\end{array}$ & $\begin{array}{l}\text { Planta } \\
\text { entera }\end{array}$ & $\begin{array}{l}\text { Verde, } \\
\text { amarillo }\end{array}$ & & $\begin{array}{l}\text { Lana de } \\
\text { alpaca }\end{array}$ & $\begin{array}{l}\text { Cusco, } \\
\text { Pampaphalla } \\
\text {, Sicuani }\end{array}$ & JAC-19314 \\
\hline $\begin{array}{l}\text { EUPHORBIACEAE } \\
\text { Alchornea } \\
\text { triplinervia (Spreng.) } \\
\text { Müll.Arg. }\end{array}$ & Zancudo caspi & $\begin{array}{l}\text { Tallos, } \\
\text { hojas, } \\
\text { corteza }\end{array}$ & Negro & --- & $\begin{array}{l}\text { lana de } \\
\text { alpaca }\end{array}$ & $\begin{array}{l}\text { Cusco, } \\
\text { Santo Tomás } \\
\text { - Río Nanay, } \\
\text { Loreto }\end{array}$ & CD-13117 \\
\hline $\begin{array}{l}\text { FABACEAE } \\
\text { Caesalpinia spinosa } \\
\text { (Molina) Kuntze }\end{array}$ & Tara & $\begin{array}{l}\text { Tallos } \\
\text { hojas }\end{array}$ & $\begin{array}{l}\text { Plomo } \\
\text { aceituno }\end{array}$ & & $\begin{array}{l}\text { Lana de } \\
\text { oveja }\end{array}$ & $\begin{array}{l}\text { Cusco, } \\
\text { Ccolcatuna, } \\
\text { Sicuani }\end{array}$ & JAC-19210 \\
\hline
\end{tabular}


Anexo 2. Especies tintóreas usadas en comunidades andinas y amazónicas peruanas.

\begin{tabular}{|c|c|c|c|c|c|c|c|}
\hline Familia/ Género/ Especie & $\begin{array}{l}\text { Nombre } \\
\text { vernáculo }\end{array}$ & $\begin{array}{l}\text { Órgano } \\
\text { empleado }\end{array}$ & $\begin{array}{l}\text { Color } \\
\text { obtenido }\end{array}$ & $\begin{array}{l}\text { Código } \\
\text { Munsell }\end{array}$ & $\begin{array}{l}\text { Tipo de } \\
\text { fibra } \\
\text { utilizada }\end{array}$ & $\begin{array}{l}\text { Región I } \\
\text { Comunidad } \\
\text { que lo } \\
\text { aplica I } \\
\text { Localidad } \\
\end{array}$ & Exsiccatae \\
\hline $\begin{array}{l}\text { Indigofera suffructicosa } \\
\text { Mill. }\end{array}$ & Añil, jango & Hojas & Marrón & 10YR3/4 & Chambira & $\begin{array}{l}\text { Loreto, } \\
\text { Amazonas, } \\
\text { Loreto }\end{array}$ & CD-13147 \\
\hline $\begin{array}{l}\text { Lupinus lindleyanus J. } \\
\text { Agardh }\end{array}$ & Taya & Hojas & $\begin{array}{l}\text { Marrón } \\
\text { canela }\end{array}$ & & $\begin{array}{ll}\text { Lana de } \\
\text { oveja }\end{array}$ & $\begin{array}{l}\text { Ancash, } \\
\text { Chunya, } \\
\text { Huaylas }\end{array}$ & JAC-19188 \\
\hline $\begin{array}{l}\text { Senna birostris (Vogel) } \\
\text { H.S.Irwin \& Barneby }\end{array}$ & Mutuy & $\begin{array}{l}\text { Tallos y } \\
\text { hojas }\end{array}$ & Verde & & $\begin{array}{l}\text { Lana de } \\
\text { alpaca }\end{array}$ & $\begin{array}{l}\text { Cusco, } \\
\text { Pampaphalla } \\
\text {, Sicuani }\end{array}$ & JAC-19223 \\
\hline $\begin{array}{l}\text { Senna reticulata (Willd.) } \\
\text { H.S.Irwin \& Barneby }\end{array}$ & Retama & Hojas & Verde & $7.5 \mathrm{GY} 4 / 4$ & Chambira & $\begin{array}{l}\text { Loreto, } \\
\text { Jenaro } \\
\text { Herrera, } \\
\text { Loreto }\end{array}$ & CD-13128 \\
\hline $\begin{array}{l}\text { Senna versicolor } \text { (Vogel } \\
\text { ) H.S.Irwin \& Barneby }\end{array}$ & Wishllac & $\begin{array}{l}\text { Tallos y } \\
\text { hojas }\end{array}$ & Verde claro & & $\begin{array}{ll}\text { Lana de } \\
\text { oveja }\end{array}$ & $\begin{array}{l}\text { Ancash, } \\
\text { Quebrada de } \\
\text { Sokup, } \\
\text { Huaylas }\end{array}$ & JAC-19320 \\
\hline GERANIACEAE & & & & & & & \\
\hline Geranium filipes Killip & & $\begin{array}{l}\text { Planta } \\
\text { entera }\end{array}$ & Rojizo & & $\begin{array}{l}\text { Lana de } \\
\text { alpaca }\end{array}$ & $\begin{array}{l}\text { Cusco, } \\
\text { Ccolcatuna, } \\
\text { Sicuani }\end{array}$ & JAC-19211 \\
\hline $\begin{array}{l}\text { Pelargonium } \\
\text { x hortorum L.H. Bailey }\end{array}$ & & Hojas & Plomo claro & & $\begin{array}{l}\text { Lana de } \\
\text { alpaca }\end{array}$ & $\begin{array}{l}\text { Cusco } \\
\text { Pampaphalla } \\
\text {, Sicuani }\end{array}$ & JAC-19229 \\
\hline $\begin{array}{l}\text { JUGLANDACEAE } \\
\text { Juglans } \quad \text { neotropica }\end{array}$ & Nogal & Hojas & Marrón & 7.5YR $2 / 4$ & Lana de & Cusco, & CD-13096, \\
\hline Diels. & & & & & alpaca & $\begin{array}{l}\text { Pampamaca, } \\
\text { San Miguel } \\
\text { de Aco }\end{array}$ & JAC 19292 \\
\hline LOGANIACEAE & & & & & & & \\
\hline Buddleja coriacea Remy & Colle & $\begin{array}{l}\text { Tallos, } \\
\text { hojas }\end{array}$ & $\begin{array}{l}\text { Marrón } \\
\text { oscuro, verde }\end{array}$ & $7.5 \mathrm{GY} 2 / 4$ & $\begin{array}{l}\text { Lana de } \\
\text { alpaca }\end{array}$ & $\begin{array}{l}\text { Cusco y } \\
\text { Ancsh, } \\
\text { Ccolcatuna, } \\
\text { Sicuani, } \\
\text { Quipia, } \\
\text { Huaylas. }\end{array}$ & $\begin{array}{l}\text { JAC-19208 } \\
\text { CD-13111 }\end{array}$ \\
\hline $\begin{array}{l}\text { Buddleja incana Ruiz \& } \\
\text { Pav }\end{array}$ & Colle & $\begin{array}{l}\text { Flores, } \\
\text { ramas }\end{array}$ & $\begin{array}{l}\text { Amarillo, } \\
\text { verde }\end{array}$ & $5 Y 8 / 10$ & $\begin{array}{l}\text { Lana de } \\
\text { oveja }\end{array}$ & $\begin{array}{l}\text { Ancash, } \\
\text { Quipia, } \\
\text { Huaylas }\end{array}$ & $\begin{array}{l}\text { JAC- } 19319 \\
\text { CD-13103 }\end{array}$ \\
\hline $\begin{array}{l}\text { MALPIGUIACEAE } \\
\text { Byrsonima stipulina J.F. } \\
\text { Macbr. }\end{array}$ & Sin referencia & Flores & $\begin{array}{l}\text { Amarillo } \\
\text { mostaza }\end{array}$ & --- & Chambira & $\begin{array}{l}\text { Cusco, } \\
\text { Santo Tomás } \\
\text { - Río Nanay, } \\
\text { Loreto }\end{array}$ & CD-13118 \\
\hline $\begin{array}{l}\text { MALVACEAE } \\
\text { Malva assurgentiflora ( } \\
\text { Kellogg) M.F. Ray }\end{array}$ & Malva & Hojas & $\begin{array}{l}\text { Marrón } \\
\text { canela }\end{array}$ & & $\begin{array}{l}\text { Lana de } \\
\text { alpaca }\end{array}$ & $\begin{array}{l}\text { Cusco, } \\
\text { Pampaphalla } \\
\text {, Sicuani }\end{array}$ & JAC-19228 \\
\hline $\begin{array}{l}\text { MELASTOMATACEAE } \\
\text { Bellucia } \\
\text { Naudin. }\end{array}$ & Sacha níspero & Hojas & $\begin{array}{l}\text { Marrón } \\
\text { canela }\end{array}$ & & Chambira & $\begin{array}{l}\text { Loreto, } \\
\text { Santo Tomás } \\
\text { - Río Nanay, } \\
\text { Loreto }\end{array}$ & CD-13116 \\
\hline $\begin{array}{l}\text { Miconia aff. prasina } \\
\text { (Sw.) DC. }\end{array}$ & Rifari & Hojas & Marrón rojizo & $7.5 \mathrm{YR} 3 / 4$ & Chambira & $\begin{array}{l}\text { Loreto, } \\
\text { Jenaro } \\
\text { Herrera, } \\
\text { Loreto } \\
\end{array}$ & CD-13126 \\
\hline
\end{tabular}


Anexo 2. Especies tintóreas usadas en comunidades andinas y amazónicas peruanas.

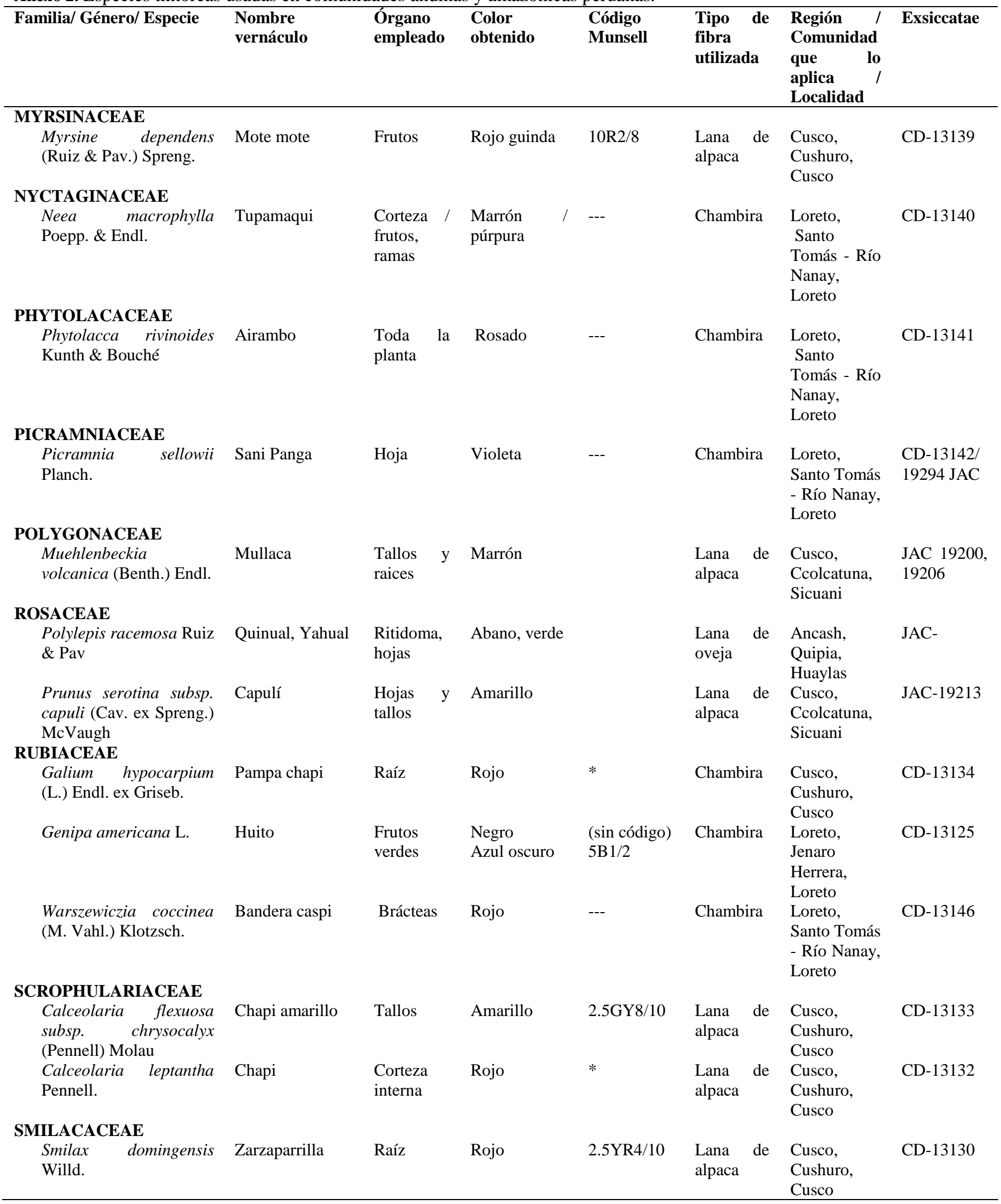


TINTES VEGETALES DE LAS COMUNIDADES ANDINAS Y AMAZÓNICAS PERUANAS

Enero - Julio 2018

Anexo 2. Especies tintóreas usadas en comunidades andinas y amazónicas peruanas.

\begin{tabular}{|c|c|c|c|c|c|c|c|}
\hline Familia/ Género/ Especie & $\begin{array}{l}\text { Nombre } \\
\text { vernáculo }\end{array}$ & $\begin{array}{l}\text { Órgano } \\
\text { empleado }\end{array}$ & $\begin{array}{l}\text { Color } \\
\text { obtenido }\end{array}$ & $\begin{array}{l}\text { Código } \\
\text { Munsell }\end{array}$ & $\begin{array}{l}\text { Tipo de } \\
\text { fibra } \\
\text { utilizada }\end{array}$ & $\begin{array}{l}\text { Región / } \\
\text { Comunidad } \\
\text { que lo } \\
\text { aplica / } \\
\text { Localidad }\end{array}$ & Exsiccatae \\
\hline $\begin{array}{l}\text { SOLANACEAE } \\
\text { Solanum nitidum Ruiz \& } \\
\text { Pav. }\end{array}$ & $\begin{array}{l}\text { Nununga, } \\
\text { nunuma }\end{array}$ & Hojas & Verde & & $\begin{array}{l}\text { Lana de } \\
\text { alpaca }\end{array}$ & $\begin{array}{l}\text { Cusco, } \\
\text { Pampaphalla }\end{array}$ & JAC-19219 \\
\hline $\begin{array}{l}\text { URTICACEAE } \\
\quad \text { Urtica flabellata } \text { Kunth }\end{array}$ & Ortiga & $\begin{array}{l}\text { Toda la } \\
\text { planta }\end{array}$ & Verde & $5 \mathrm{GY} 2 / 2$ & $\begin{array}{l}\text { Lana de } \\
\text { alpaca }\end{array}$ & $\begin{array}{l}\text { Cusco, } \\
\text { Pampamaca, } \\
\text { San Miguel } \\
\text { de Aco }\end{array}$ & CD-13091 \\
\hline $\begin{array}{l}\text { ZINGIBERACEAE } \\
\text { Curcuma longa } \mathrm{L} .\end{array}$ & Guisador, palillo & Rizomas & Amarillo & $7.5 \mathrm{YR} 5 / 10$ & Chambira & $\begin{array}{l}\text { Loreto, } \\
\text { Jenaro } \\
\text { Herrera, } \\
\text { Loreto }\end{array}$ & CD-13122 \\
\hline $\begin{array}{l}\text { Renealmia alpinia } \\
\text { (Rottb.) Maas. }\end{array}$ & Mishquipanga & Frutos & Morado & --- & Chambira & $\begin{array}{l}\text { Loreto, } \\
\text { Santo Tomás } \\
\text { - Río Nanay, } \\
\text { Loreto }\end{array}$ & CD-13143 \\
\hline $\begin{array}{l}\text { Renealmia thyrsoidea } \\
\text { (Ruiz \& Pav.) Poepp. \& } \\
\text { Endl. }\end{array}$ & Mishquipanga & Frutos & Morado & $2.5 \mathrm{YR} 1 / 4$ & Chambira & $\begin{array}{l}\text { Loreto, } \\
\text { Jenaro } \\
\text { Herrera, } \\
\text { Loreto }\end{array}$ & CD-13127 \\
\hline
\end{tabular}

JAC= Joaquina Albán, $\mathrm{CD}=$ Camilo Díaz.

${ }^{1}$ Departamento de Etnobotánica. Museo de Historia Natural. Universidad Nacional Mayor de San Marcos. jalbanc@unmsm.edu.pe.

${ }^{2}$ Laboratorio de Botánica Aplicada. Laboratorios de Investigación y Desarrollo. Facultad de Ciencias y Filosofía. Universidad Peruana Cayetano Heredia.

${ }^{3}$ Unidad de Investigación en Productos Naturales, Laboratorios de Investigación y Desarrollo. Facultad de Ciencias y Filosofía. Universidad Peruana Cayetano Heredia. 